\title{
The effect of anatomic differences on the relationship between renal artery and diaphragmatic crus
}

\author{
K. Esen ${ }^{1}$, S. Tok ${ }^{1}$, Y. Balci ${ }^{1}$, F.D. Apaydin'1 , E. Kara ${ }^{1}$, D. Uzmansel ${ }^{2}$ \\ ${ }^{1}$ Department of Radiology, Mersin University, School of Medicine, Mersin, Turkey \\ 2Department of Anatomy, Mersin University, School of Medicine, Mersin, Turkey
}

[Received: 9 July 2017; Accepted: 27 July 2017]

Background: The aim of this study is to investigate the effect of anatomic differences on the relationship between renal artery and diaphragmatic crus via the touch of two structures.

Materials and methods: The study included dynamic computed tomography (CT) scans of 308 patients performed mainly for characterisation of liver and renal masses. Anatomic differences including the thickness of the diaphragmatic crus, the localisation of renal artery ostium at the wall of aorta, the level of renal artery origin with respect to superior mesenteric artery were evaluated. Statistical relationships between renal artery-diaphragmatic crus contact and the anatomic differences were assessed.

Results: Thickness of the diaphragmatic crus at the level of renal artery origin exhibited a statistically significant relationship to renal artery-diaphragmatic crus contact at the left $(p<0.001)$ and right side $(p<0.001)$. There was a statistically significant relationship between high renal artery origin and renal artery-diaphragmatic crus contact at the left $(p<0.001)$ and right side $(p=0.01)$. The localisation of renal artery ostium at the wall of aorta (right side, $p=0.436$, left side, $p=0.681$ ) did not demonstrate a relationship to renal artery-diaphragmatic crus contact.

Conclusions: Thickness of the diaphragmatic crus and high renal artery origin with respect to superior mesenteric artery are crucial anatomic differences determining the relationship of renal artery and diaphragmatic crus. (Folia Morphol 2018; 77, 1: 22-28)

Key words: entrapment, diaphragmatic crus, renal artery

\section{INTRODUCTION}

Variations of renal artery and the anatomy of diaphragmatic crus have been well-defined [12, 17]. However, anatomic differences and variations may cause an abnormal relationship between renal ar- tery and diaphragmatic crus which is a rare cause of renal artery stenosis. This relationship, causing renal artery stenosis, has been described as renal artery entrapment syndrome (RAES) and evaluated in earlier reports $[5,9,19,20]$. The fibres forming part of the 
crus of the diaphragm or psoas muscle impinging on the renal artery cause compression and narrowing of renal artery lumen in RAES [21]. Although, the entrapment is usually observed at the ostium of the artery, it may also be truncal $[21,23]$. Renal artery descending along the aorta and a short ostial stenosis in a patient free of atheroma were suggestive of renal artery entrapment [23]. Congenital abnormalities such as abnormal musculo-tendinous fibres, high ectopic renal artery origin or hypertrophic diaphragmatic crus were found to be responsible for these entrapments $[2,23]$. Whereas, the compression of renal artery with diaphragmatic crus is not mentioned as congenital and it has been reported that entrapments may develop with the alterations between the relationship of renal artery and diaphragmatic crus over time [23]. It seems difficult to predict which patients will develop RAES; nevertheless, a close relationship or contact between renal artery and diaphragmatic crus may be a predisposition. Scoliosis, tortuosity of aorta, degenerative changes of vertebrae, retroperitoneal masses may also be effective. Our aim is to evaluate the relationship of renal artery and diaphragmatic crus via the contact between two structures and to investigate the anatomical differences that may affect this relationship such as the thickness of the crus at the level of renal artery origin, localisation of renal artery ostium at the wall of aorta and level of renal artery origin with respect to superior mesenteric artery (SMA).

\section{MATERIALS AND METHODS}

Patients, admitted to our clinic for dynamic computed tomography (CT) examination between January 2014 and February 2016 were retrospectively evaluated. Dynamic CT examinations were performed mainly for the characterisation of liver or renal masses. Patients with nephrectomies, retroperitoneal masses affecting renal arteries, ectopic kidneys, scoliosis, aortic aneurysms were excluded. Examinations with inadequate image quality were also excluded. The study group consisted of 308 patients after the exclusion. Approval for the study was obtained from the ethical committee of our institution (date of approval: 2016, number of decision: 2016/64).

Multiphasic CT scans were performed using a 64-slice scanner (Toshiba Aquilion 64, Toshiba Medical Systems, Tokyo, Japan). For each examination $90 \mathrm{cc}$ nonionic contrast material was administered through an antecubital venous catheter with an aver- age rate of $2-3 \mathrm{~mL} / \mathrm{s}$. Arterial, portal and late phase images were obtained with a slice thickness of $0.5 \mathrm{~mm}$. The abdomen was scanned at supine position with inspiratory breath-holding for each phase and arterial phase images were used for the evaluation. The timing of arterial phase images was $25 \mathrm{~s}$ and $40 \mathrm{~s}$ for liver and renal masses, respectively. In each case, the images were evaluated in consensus by two experienced radiologists on axial and sagittal images. Multiplanar reformatted reconstruction (MPR), maximum intensity projection (MIP) images were obtained from axial images.

Totally 308 patients (male/female: 177/131; mean age $57.4 \pm 14.1$ years, range 10-89 years) were included in this retrospective study.

The thickness of the diaphragmatic crus was evaluated on axial images at the level of renal artery for each side. The measurements were made at the thickest part in a plane perpendicular to the long axis of the crura as in the study reported by Brengle et al. [3] The levels of renal artery origins were evaluated depending on the level of SMA. In order to evaluate with respect to SMA origin, the height of vertebrae corpus was divided into five segments on sagittal images and disc space was accepted as one segment. The level of SMA and renal artery origins were determined with respect to this segmentation. Renal arteries originating at the same level, higher level or only one segment lower level compared to SMA were accepted as high origin.

Figure 1 demonstrates the segmentation of aorta on axial images. Aorta was divided into four quarters on axial images and the ostium localisation of renal artery was also noted as anterior, midline and posterior at each side.

Finally, the contact of the crus and renal artery was assessed and the contact at only one point was enough to accept positive. In addition to the measurements, the examiners noted the compression of the lumen of main or accessory renal arteries by the crus, if there was any.

\section{Statistical analysis}

All analyses were performed using IBM SPSS Statistics Version 20.0 statistical software package. Categorical variables were expressed as numbers and percentages, whereas continuous variables were summarised as mean and standard deviation. Chi-square test was used to compare categorical variables between the groups. For comparison of con- 


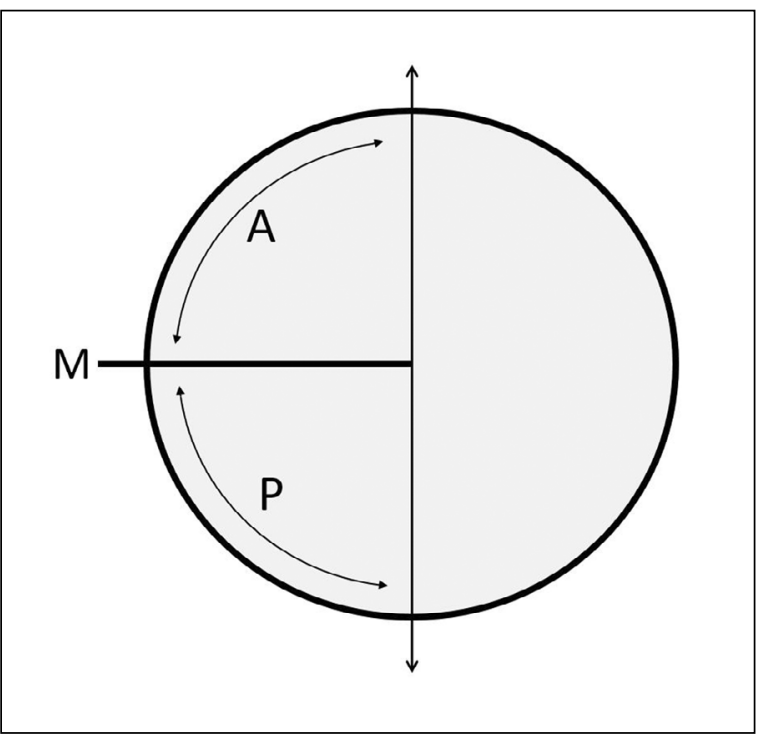

Figure 1. The schematic division of lateral half of the aorta in order to evaluate the localisation of renal artery ostium $(\mathrm{A}$ - anterior; $\mathrm{M}$ - midline; $\mathrm{P}$ - posterior). Note that the midline is limited to the lateral midpoint of aorta.

Table 1. The thickness of right and left crus

\begin{tabular}{llc}
\hline & \multicolumn{2}{c}{ Thickness [mm] } \\
\cline { 2 - 3 } & $\begin{array}{c}\text { Mean } \pm \text { standard } \\
\text { deviation }\end{array}$ & $\begin{array}{c}\text { Range } \\
{[\mathrm{mm}]}\end{array}$ \\
\hline Right crus & $9.4 \pm 2.9$ & $2-19.1$ \\
Left crus & $3.2 \pm 3.7$ & $0-15.8$ \\
\hline
\end{tabular}

Table 2. Distribution of the patients according to the contact between diaphragmatic crus and renal artery at the level of renal artery origins

\begin{tabular}{lcc}
\hline & Group 1 & Group 2 \\
\hline Right & $121(39.3 \%)$ & $187(60.7 \%)$ \\
Left & $48(15.6 \%)$ & $260(84.4 \%)$ \\
\hline
\end{tabular}

Group 1 - Patients with contact between renal artery and diaphragmatic crus.

Group 2 - Patients that have no contact between renal artery and diaphragmatic crus. tinuous variables between two groups, the Student's t-test was used. The statistical level of significance for all tests was considered to be 0.05 .

\section{RESULTS}

The range of the thickness and mean thickness of diaphragmatic crus is shown in Table 1. The mean thickness of the right diaphragmatic crus was higher than that of the left one. We also could not observe the left diaphragmatic crus in $50.6 \%$ of the patients at the level of renal artery origin.

Table 2 demonstrates the distribution of the patients according to the contact between diaphragmatic crus and renal artery at the level of renal artery origins. The number of the patients that have no contact between diaphragmatic crus and renal artery was higher than the patients that have a contact at the left and right. Table 3 shows the mean thickness of right and left diaphragmatic crus of the patients according to the contact between diaphragmatic crus and renal artery at the level of renal artery origins. The mean thickness of diaphragmatic crus that contact with renal artery was higher than the mean thickness of crus that does not contact with renal artery at both sides. There was a statistically significant relationship between the thickness of the crus and the contact at the left $(p<0.001)$ and right $(p<0.001)$.

The mean age of the patients who have a contact between renal artery and diaphragmatic crus at the right and left side was $52.5 \pm 14$ and $49 \pm 14.5$, respectively. The mean age of the patients whose renal artery was not in contact with diaphragmatic crus were $60.5 \pm 13.3$ at the right side and $58.9 \pm$ \pm 13.5 at the left side. We demonstrated a statistically significant relationship between age and contact at the left $(p<0.001)$ and right $(p<0.001)$ side. As a result, the mean age of the patients that do not have a contact between diaphragmatic crus and renal artery were higher than the patients having a contact.

Table 3. The mean thickness of right and left diaphragmatic crus of the patients according to the contact between diaphragmatic crus and renal artery at the level of renal artery origins

\begin{tabular}{lccc}
\hline & \multicolumn{3}{c}{ Mean thickness [mm] \pm standard deviation } \\
\cline { 2 - 4 } & Group 1 & Group 2 & p \\
\hline Right crus & $10.7 \pm 2.8$ & $8.5 \pm 2.7$ & $<0.001$ \\
Left crus & $8.2 \pm 2.5$ & $2.3 \pm 3.2$ & $<0.001$ \\
\hline
\end{tabular}

Group 1 - Patients with contact between renal artery and diaphragmatic crus.

Group 2 - Patients that have no contact between renal artery and diaphragmatic crus. 
Table 4. Distribution of the right and the left renal artery origins according to the localization of ostium at the wall of aorta

\begin{tabular}{lcc}
\hline \multirow{2}{*}{$\begin{array}{l}\text { Localisation } \\
\text { of ostium }\end{array}$} & \multicolumn{2}{c}{ Renal artery } \\
\cline { 2 - 3 } & Right & Left \\
\hline Anterior & $222(72.1 \%)$ & $23(7.5 \%)$ \\
Midline & $86(27.9 \%)$ & $190(61.7 \%)$ \\
Posterior & $0(0 \%)$ & $95(30.8 \%)$ \\
\hline
\end{tabular}

Table 4 shows the distribution of right and left renal artery origins with respect to the localisation of ostium at the wall of aorta on axial images. Although the majority of right renal arteries were originating from the anterior aspect of the aorta, there was not any right renal artery originating from the posterior aspect. Whereas, $30.8 \%$ of left renal artery were arising from the posterior aspect of aorta.

The relationship between high renal artery origin with respect to SMA and renal artery-diaphragmatic crus contact was statistically significant at the right $(p=0.01)$ and the left $(p<0.001)$ side. Among the patients that have right renal artery-diaphragmatic crus contact, the percentage of high and normal right renal artery origins were $51.1 \%$ and $34.5 \%$, respectively. On the other hand, among the patients that have left renal artery-diaphragmatic crus contact, the percentage of high and normal left renal artery origins were $41.3 \%$ and $11.1 \%$, respectively.
There was not a statistically significant relationship between the localisation of renal artery origins at the wall of aorta and renal artery-diaphragmatic crus contact (right side, $p=0.436$; left side, $\mathrm{p}=0.681)$.

Totally, 54 (17.5\%) patients had one or more accessory renal artery. Twelve accessory renal arteries were in touch with the crus of diaphragm. Compression of the main renal artery by the diaphragmatic crus was detected in 3 patients with stenosis lower than $40 \%$ (Figs. 2, 3).

\section{DISCUSSION}

As mentioned before, abnormal musculotendinous fibres, high ectopic renal artery origin, and hypertrophic diaphragmatic crus were reported as the main responsible factors for entrapment syndrome in various studies $[6,10,19,22,23]$. In the study reported by Thony et al. [23], the diagnostic and therapeutic approaches to RAES were evaluated in 15 patients. Arazińska et al. [2] investigated the anatomical factors that may be related to the entrapment of renal artery in 7 patients. In cases of entrapments, the relationship is observed as a truncal or ostial compression that causes different degrees of stenosis [23]. In the present study, we accepted the contact of two structures without any compression as an indicator of relationship different from RAES. However, we did not take the length and the localisation of contact into account.

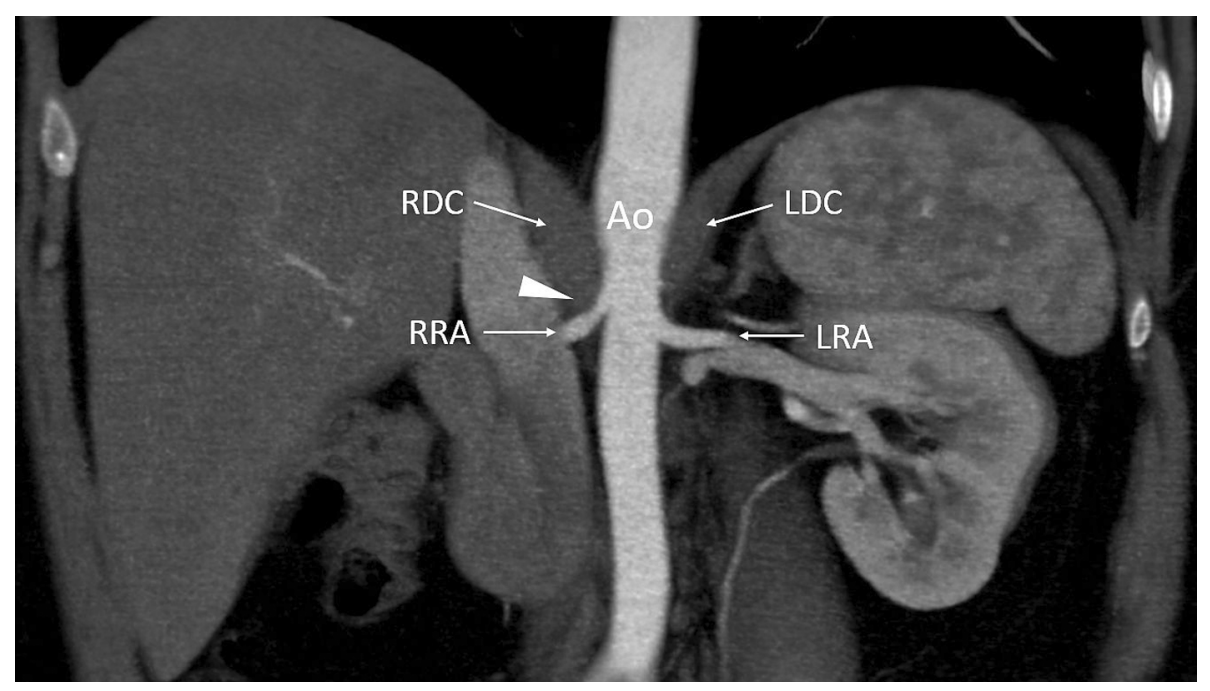

Figure 2. Coronal volume rendered computed tomography image showing the minimal compression of right renal artery with the crus of the diaphragm (arrowhead); Ao — aorta; RRA — right renal artery; LRA — left renal artery; RDC — right diaphragmatic crus; LDC — left diaphragmatic crus. 


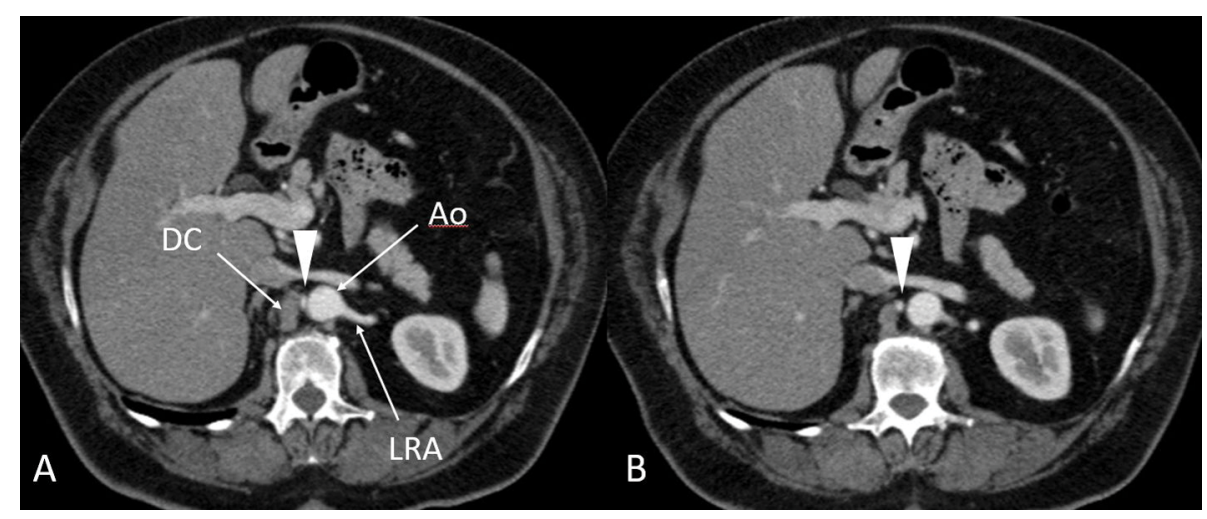

Figure 3. Axial consecutive computed tomography images. A, B. Ostial compression of the right main renal artery with the right crus of the diaphragm. Note that the compression is observed only at the ostium of renal artery (arrowheads); Ao — aorta; DC — diaphragmatic crus; LRA — left renal artery.

The diaphragm forms between the $4^{\text {th }}$ and $12^{\text {th }}$ weeks of embryonic life [17]. The septum transversum and pleuroperitoneal membranes fuse with the dorsal mesentery of the oesophagus which constitutes the median portion of the diaphragm [15]. The crura of the diaphragm develop from myoblasts that grow into the dorsal mesentery of the oesophagus [15]. While the tendinous aspect of the right crus attaches to the ventral surface of the lumbar vertebral bodies and to the intervertebral fibrocartilage of the first three lumbar vertebrae, the left crus attaches to the first two lumbar vertebral bodies [17]. The right crus is longer and broader than the left [17]. In the present study, the mean thickness of right crus was higher than the left crus at the level of renal artery origins. However, we could not observe the left diaphragmatic crus in $50.6 \%$ of the patients at the level of renal artery origins.

The kidneys ascend to the lumbar region between the $6^{\text {th }}$ and $9^{\text {th }}$ weeks of the embryo [13]. During the cephalic migration of the kidneys, they are vascularised by a succession of transient aortic sprouts that arise at higher levels progressively [13]. The lowest suprarenal artery develops into the main renal artery from the middle group, while more caudal and cephalic vessels degenerate [2]. The final pair of arteries, most commonly, originate from the lower margin of the L1 vertebra on the right side and from the upper margin of the L2 vertebra on the left side $[7,13]$. Rarely, renal arteries may originate from the more proximal portion of the abdominal aorta above the origin of the SMA [12]. The persistence of cranial group lateral mesonephric arteries is thought to be re- lated to the formation of a renal artery that branches above the coeliac trunk [7].

High renal artery origin seems to be another factor in renal artery-diaphragmatic crus relationship. Arazińska et al. [2] revealed that the most important anatomical risk factors of renal artery stenosis are level of renal artery origin in respect to vertebrae and distance between main left and right renal arteries. Generally, renal arteries arise below the origin of the SMA. Herein, we evaluated the level of renal artery origin with respect to SMA instead of lumbar vertebrae and observed higher contact ratios in the patients with higher originated renal artery.

The significance of the localisation of renal artery ostium at the level of origin has not been described in entrapment syndromes. We did not demonstrate a relationship between the ostium localisation and contact. However, the majority $(72.1 \%)$ of the right renal arteries originated at the anterior aspect of aorta and $61.7 \%$ of the left renal artery originated at the midline according to our classification. It has been described that right renal artery courses anterior at first 1-2 cm and then turns posterior [16]. The left renal artery has a more linear course. To the best of our knowledge there is not enough data about the aetiology of this course. We observed a curving in the course of right renal artery around the crus with a broad-based contact as if the renal artery is displaced by the crus in some of the patients. However, majority of the patients demonstrated the similar curving course without any contact and there was fat tissue between the renal artery and crus (Fig. 4). In a study performed with children, Brengle et al. [3] 


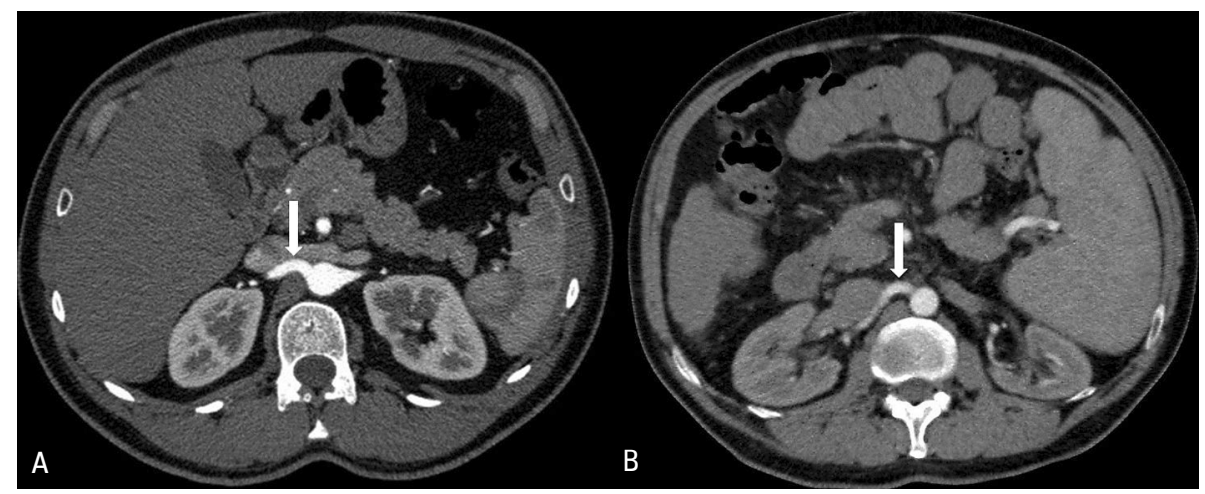

Figure 4. Axial computed tomography images demonstrate the curving of right renal artery at different patients. A. Thirty-seven-year-old man who is a liver donor. The right renal artery has a broad-based contact with diaphragmatic crus (arrow). B. Sixty-year-old man with liver cirrhosis. Note the fat tissue between diaphragmatic crus and renal artery without any contact at curving point (arrow).

reported that crural width does not increase with age and the diaphragmatic crus of smaller children appear relatively large. Moes et al. [14] observed that diaphragmatic crus in the neonate are considerably enlarged in comparison with adults. As the body grows, crus will occupy a smaller area compared with other organs during the childhood. On the other hand, Caskey et al. [4] described the evolution of diaphragm with aging and demonstrated that diaphragm muscle thickness did not change significantly with increasing age. In the study reported by Dovgan et al. [8], maximal crural thickness occurred at the origin of SMA in $73 \%$ of patients and the range of maximal crural thickness was $1.8-18.8 \mathrm{~mm}$ in men and $1.8-21.1 \mathrm{~mm}$ in women which remained constant from the second to the $8^{\text {th }}$ decades of life. In our study group, the mean age of the patients who do not have a contact between diaphragmatic crus and renal artery were higher than the patients having a contact. However, our study population was not homogeneous and most of the patients were at $5^{\text {th }}$ and $6^{\text {th }}$ decades of life. Further analyses are needed about the effects of aging on the relationship between renal artery and diaphragmatic crus which may also be helpful to understand the mechanism of RAES. In consequence, diaphragmatic crus may be the reason of renal artery curving with a close relationship during development and this relationship between two structures might decrease physiologically by the time during childhood.

The accessory renal arteries constitute the most common renal artery variations [12]. In addition to the main renal artery, the contact of diaphragmatic crus to accessory renal arteries or compression of the artery by the crus may be seen and cause arterial stenosis. In our study, there was more than one renal artery in $17.5 \%$ of the patients and $22 \%$ of accessory renal arteries were in contact with diaphragmatic crus. Accessory renal arteries of 3 patients were compressed minimally with diaphragmatic crus which were not causing hypertension. The clinical significance of accessory renal artery stenosis is controversial. Gupta et al. [11] revealed that stenosis in accessory renal arteries is not a risk factor for hypertension. Saba et al. [18] did not find any statistical association between the presence of accessory renal artery stenosis and hypertension. However, Saba et al. [18] also advised that accessory renal artery stenosis is an important finding which should prompt the radiologist to evaluate its possible association with hypertension.

Renal artery entrapment was found in $0.97 \%$ of our study population. However, the degree of stenosis was lower than $40 \%$ and the patients did not have hypertension. Entrapments were at the right side in 2 patients and at the left in 1 patient. The thickness of the right crus was $13.6 \mathrm{~mm}$ and $12.2 \mathrm{~mm}$ in 2 patients while the thickness of left crus was $8.2 \mathrm{~mm}$ in the patient with left entrapment. None of the patients had high localised renal artery with respect to SMA. All patients were over 62 years old.

\section{Limitations of the study}

It should be noted that the study has some limitations. First of all, this is a retrospective study; thus, neither the examinations were standardised nor were the patients randomised. Earlier reports demonstrated that the thickness of the crus increases during 
inspiration [1]. All phases of dynamic CT in our study were taken during inspiration so that measurements have been made in the thickest form of the crus and the relationship may have been overestimated. We did not evaluate the body mass index of the patients which may affect the thickness of the diaphragmatic crus or retroperitoneal fat tissue.

\section{CONCLUSIONS}

In conclusion, this study allowed us to investigate the possible anatomic differences affecting the diaphragmatic crus-renal artery relationship at the level of renal artery origins in a larger population. Thickness of the diaphragmatic crus and high renal artery origin with respect to SMA determine the relationship of renal artery and diaphragmatic crus. However, it is difficult to predict the development of renal artery entrapment via the contact of two structures.

\section{REFERENCES}

1. Anda S, Røysland P, Fougner R, et al. CT appearance of the diaphragm varying with respiratory phase and muscular tension. J Comput Assist Tomogr. 1986; 10(5): 744-745, indexed in Pubmed: 3745542.

2. Arazińska A, Polguj M, Topol M, et al. Renal artery entrapment - anatomical risk factors rating. Folia Morphol. 2016; 75(4): 486-492, doi: 10.5603/FM.a2016.0017, indexed in Pubmed: 27830874.

3. Brengle M, Cohen MD, Katz B. Normal appearance and size of the diaphragmatic crura in children: CT evaluation. Pediatr Radiol. 1996; 26(11): 811-814, indexed in Pubmed: 8929383.

4. Caskey $\mathrm{Cl}$, Zerhouni EA, Fishman EK, et al. Aging of the diaphragm: a CT study. Radiology. 1989; 171(2): 385-389, doi: 10.1148/radiology.171.2.2704802, indexed in Pubmed: 2704802.

5. D'abreu Stricland B. Developmental renal-artery stenosis. Lancet. 1962; 2(7255): 517-521, indexed in Pubmed: 13859087.

6. Déglise S, Corpataux JM, Haller C, et al. Bilateral renal artery entrapment by diaphragmatic crura: a rare cause of renovascular hypertension with a specific management. J Comput Assist Tomogr. 2007; 31(3): 481-484, doi: 10.1097/01.rct.0000250114.72338.5a, indexed in Pubmed: 17538300.

7. Delasotta LA, Olivieri B, Malik A, et al. Thoracic renal artery: a rare variant. A case study and literature review. Surg Radiol Anat. 2015; 37(5): 561-564, doi: 10.1007/s00276014-1379-2, indexed in Pubmed: 25270524.

8. Dovgan DJ, Lenchik L, Kaye AD. Computed tomographic evaluation of maximal diaphragmatic crural thickness. Conn Med. 1994; 58(4): 203-206, indexed in Pubmed: 8045119.

9. Dure-Smith P, Bloch RD, Fymat AL, et al. Renal artery entrapment by the diaphragmatic crus revealed by heli- cal CT angiography. AJR Am J Roentgenol. 1998; 170(5): 1291-1292, doi: 10.2214/ajr.170.5.9574603, indexed in Pubmed: 9574603.

10. Gaebel G, Hinterseher I, Saeger HD, et al. Compression of the left renal artery and celiac trunk by diaphragmatic crura. J Vasc Surg. 2009; 50(4): 910-914, doi: 10.1016/j. jvs.2009.05.004, indexed in Pubmed: 19786242.

11. Gupta A, Tello R. Accessory renal arteries are not related to hypertension risk: a review of MR angiography data. AJR Am J Roentgenol. 2004; 182(6): 1521-1524, doi: 10.2214/ ajr.182.6.1821521, indexed in Pubmed: 15150000.

12. Hazırolan T, Öz M, Türkbey B, et al. CT angiography of the renal arteries and veins: normal anatomy and variants. Diagn Interv Radiol. 2011; 17(1): 67-73, doi: 10.4261/13053825.DIR.2902-09.1, indexed in Pubmed: 20151356.

13. He B, Hamdorf JM. Clinical importance of anatomical variations of renal vasculature during laparoscopic donor nephrectomy. OA Anatomy. 2013; 1(3), doi: 10.13172/20527829-1-3-865.

14. Moes MJ, Filly RA. The neonatal diaphragmatic crura are hypertrophied: a necessary preparation for the first breath? J Ultrasound Med. 2003; 22(7): 715-718, indexed in Pubmed: 12862271.

15. Moore KL, Persaud T, Torchia MG. Body cavities and diaphragm. In: The developing human: clinically oriented embryology. 9th ed. Philadelphia, Pa: Saunders 2013: 145-158.

16. Pozzi-Mucelli F, Pellegrin A, Pozzi-Mucelli R. Radiological Imaging of the Kidney. 2nd ed. Springer, Berlin 2014: 189-222.

17. Restrepo CS, Eraso A, Ocazionez D, et al. The diaphragmatic crura and retrocrural space: normal imaging appearance, variants, and pathologic conditions. Radiographics. 2008; 28(5): 1289-1305, doi: 10.1148/rg.285075187, indexed in Pubmed: 18794306.

18. Saba L, Sanfilippo R, Montisci R, et al. Accessory renal artery stenosis and hypertension: are these correlated? Evaluation using multidetector-row computed tomographic angiography. Acta Radiol. 2008; 49(3): 278-284, doi: 10.1080/02841850701777408, indexed in Pubmed: 18365815.

19. Shruthi BN. Unilateral entrapment of the renal artery by diaphragmatic crus. Natl J Med Res. 2013; 3: 412-413.

20. Silver D, Clements JB. Renovascular hypertension from renal artery compression by congenital bands. Ann Surg. 1976; 183(2): 161-166, indexed in Pubmed: 1247314.

21. Singham $S$, Murugasu $P$, Macintosh J, et al. Left main renal artery entrapment by diaphragmatic crura: spiral CT angiography. Biomed Imaging Interv J. 2010; 6(2): e11, doi: 10.2349/biij.6.2.e11, indexed in Pubmed: 21611033.

22. Spies JB, LeQuire MH, Robison JG, et al. Renovascular hypertension caused by compression of the renal artery by the diaphragmatic crus. Am J Roentgenol. 1987; 149(6): 1195-1196, doi: 10.2214/ajr.149.6.1195, indexed in Pubmed: 3500606.

23. Thony F, Baguet JP, Rodiere $M$, et al. Renal artery entrapment by the diaphragmatic crus. Eur Radiol. 2005; 15(9): 1841-1849, doi: 10.1007/s00330-005-2710-4, indexed in Pubmed: 15778837. 